\title{
As atitudes e o conhecimento sobre práticas de vida saudáveis de uma amostra da população de Juiz de Fora
}

\author{
José Antonio Chehuen Neto* \\ Renato Erothildes Ferreira* \\ Ana Vitoria Vitoreti Martins*** \\ Jéssica Miquelitto Gasparoni ${ }^{* * *}$ \\ Danillo Zeferino de Oliveira Souza ${ }^{* * *}$ \\ Flávia Paiva dos Santos Pereira ${ }^{* * *}$
}

\begin{abstract}
RESUMO
O novo pentáculo do bem-estar refere-se a cinco características do estilo de vida que auxiliam a promoção da saúde individual: níveis de stress, características nutricionais, atividades físicas habituais, comportamento preventivo e qualidade dos relacionamentos humanos. Ao percebermos a dificuldade na alteração do estilo de vida dos pacientes e, também, em atuar na prevenção de doenças ou no seu tratamento, buscamos identificar os obstáculos na mudança para um estilo de vida saudável, além de avaliar hábitos de vida e conhecimento sobre práticas saudáveis. Assim, elaboramos um estudo transversal, descritivo e quantitativo realizado na cidade de Juiz de Fora - MG, coletando-se dados a partir do questionário validado, novo pentáculo do bem-estar, com amostra de 462 indivíduos. A maioria dos entrevistados têm noções dos hábitos saudáveis, com 55,2\% da amostra baseando-se em orientações médicas; $93,7 \%$ acreditam nos benefícios da mudança de estilo de vida; $92,2 \%$ supõe que hábitos saudáveis atuais repercutem no futuro e 53,6\% realizam algum tipo de atividade física. De modo geral, a amostra mantém hábitos nutricionais satisfatórios, $61,9 \%$ relataram ingesta diária de 05 porções de vegetais e 53,7\% evitavam alimentos gordurosos e/ou doces. As dificuldades encontradas relacionam-se principalmente aos fatores culturais, vinculadas às crenças. Quanto as possibilidades/efetividades de mudanças; a falta de tempo foi a segunda maior dificuldade encontrada. Ao fim, concluiu-se que o perfil que melhor cuida da própria saúde compreende aqueles com nível de escolaridade mais elevado, menores de 34 anos e que recebem quatro ou mais salários mínimos por mês, independente do sexo.
\end{abstract}

Palavras-Chave Estilo de vida. Saúde. Atividade física.

\section{INTRODUÇÃO}

Estilo de vida compreende um conjunto cotidiano de padrões e ações comportamentais identificáveis, realizadas individualmente ou em grupo e que refletem os atos e os valores de uma pessoa ou grupos de pessoas. Os componentes do estilo de vida podem se alterar ao longo do tempo à medida que o indivíduo inclui ou exclui comportamentos que julga necessários para se alcançar um estilo de vida saudável (SALLIS; OWEN, 1999).

É imprescindível, ao abordar esse tema, levar em consideração o atual conceito de saúde da Organização Mundial de Saúde (OMS), dado como "o estado de completo bem-estar físico, mental e social e espiritual e não apenas a ausência de afecções ou doenças", pois é essa concepção que direciona as atuais políticas de promoção de saúde e que norteia os componentes de um estilo de vida saudável (BUSS,2002;
VASCONCELOS; SCHMALLER, 2014; WORLD HEALTH ORGANIZATION [WHO], 1946).

A mudança de hábitos de vida pouco ou nada saudáveis para atitudes saudáveis permite atuar sobre fatores clássicos associados às doenças e aos agravos não transmissíveis, tais como as doenças cardiovasculares. A mudança está subordinada a diversos fatores como: sexo, idade, etnia, estado civil, escolaridade, hábitos não aconselhados como fumo e álcool e nível socioeconômico (DOSSE et al., 2009); outros seriam determinados por particularidades da própria doença: a cronologia, a sintomatologia, a gravidade, características do tratamento, tipo de medicamento usado, custo, efeitos adversos e dieta, entre outros. Entretanto, podemos citar fatores com características mais gerais, tais como os dependentes de crenças e valores culturais (RICOU et al., 2004).

A estratégia de aconselhamento e incentivo da promoção de saúde por intervenção nos fatores de

* Universidade Federal de Juiz de Fora, Faculdade de Medicina, Departamento de Cirurgia - Juiz de Fora, MG.

** Universidade Federal de Juiz de Fora, Faculdade de Medicina, Grupo de Estudos em Cirurgia Experimental - Juiz de Fora, MG.

*** Universidade Federal de Juiz de Fora, Faculdade de Medicina - Juiz de Fora, MG. 
risco é amplamente adotada na atenção primária a saúde da população e é de fundamental importância em todo o contexto, no qual doenças conhecidas como multifatoriais atingem proporções cada vez maiores (BAUM; FISHER, 2014; NAHAS; BARROS; FRANCALACCI, 2000).

Em outras palavras, certos hábitos adotados pelos indivíduos podem acarretar o surgimento de alterações clínicas negativas, fazendo-se necessárias adequações positivas no estilo de vida para se reduzir o risco e aumentar o sucesso do tratamento. De acordo com Nahas, Barros e Francalacci (2000), em muitas doenças, a mudança do estilo de vida é proposta como vital, ora como parte do tratamento, ora como forma de se obter melhorias no quadro da doença atual ou evitar sua progressão.

$\mathrm{Na}$ última década, um novo pentáculo do bemestar é utilizado, e faz referência a cinco características do estilo de vida que auxiliam a promoção da saúde: níveis de stress, características nutricionais, atividades físicas habituais, comportamento preventivo e a qualidade dos relacionamentos humanos (NAHAS; BARROS; FRANCALACCI, 2000). Com base nestes atuais parâmetros e ao percebermos no cotidiano profissional o quão difícil é para os pacientes alterar positivamente o estilo de vida na prevenção de doenças ou efetivamente no próprio tratamento, nos motivamos a identificar as dificuldades encontradas na adoção de um estilo de vida saudável, caracterizar os hábitos de vida e avaliar o nível de conhecimento de um grupo de indivíduos sobre práticas saudáveis.

\section{Material e Métodos}

O delineamento do estudo foi do tipo transversal de base populacional e estima a prevalência da variável de desfecho, ou seja, o conhecimento acerca da temática "As práticas saudáveis e as mudanças do estilo de vida".Trata-se de uma pesquisa aplicada, de natureza quantitativa, exploratória e descritiva, realizada na cidade de juiz de Fora, Minas Gerais.

A cidade de Juiz de Fora encontra-se subdividida em cinco regiões demográficas, segundo o Censo Demográfico de 2010 (norte, sul, centro, leste e oeste). Para realização da pesquisa, foram sorteados dois setores censitários para cada uma das cinco regiões. Para garantir a homogeneidade das condições socioeconômicas, foram sorteados dois setores censitários para cada uma das cinco regiões. Um setor censitário consiste nos centros comerciais e seu entorno.

Os dados foram colhidos mediante aplicação de questionário estruturado em entrevista com delineamento face a face, e os participantes eram abordados na rua durante sua passagem, de forma cordial e padronizada por pesquisador habilitado. Após isso, foram convidados a posicionar-se fora do fluxo de pessoas, garantindo um diálogo reservado, inicialmente receberam informações detalhadas acerca do estudo e foram convidados a participar do mesmo. Para tal, assinaram o TCLE concordando de forma voluntaria com essa participação. A coleta dos dados foi realizada por três pesquisadores, devidamente identificados.

Previamente a aplicação dos questonários houve treinamento dos pesquisadores, com calibração das técnicas de medida. A calibração do instrumento visou treinar o examinador, a fim de assegurar a uniformização de interpretação, de compreensão e de aplicação dos critérios do questionário, minimizando as variações de resposta e interpretação por parte do avaliado.

Os dados foram colhidos mediante aplicação de questionário estruturado em entrevista. Os critérios de inclusão foram: ser morador da cidade de Juiz de Fora - Minas Gerais e ter idade $\geq 18$ anos. Como perda amostral definiu-se os questionários interrompidos por qualquer motivo e dados incompletos.

$\mathrm{O}$ (n) para esta pesquisa foi calculado em 500 indivíduos. O cálculo da amostra considerou $5 \%$ de erro máximo desejado para a pesquisa, para mais ou para menos, para um nível de confiança de $95 \%$, e possível nível de recusa de aproximadamente 15\%. Este espectro amostral atende rigorosamente aos critérios e as necessidades estatísticas.

Em nosso estudo, foram abordadas 560 pessoas, sendo que 60 indivíduos se recusaram a participar da entrevista (12\%). Dos 500 entrevistados, 38 se enquadraram nos critérios de perda amostral $(7,6 \%)$, contemplando uma amostra de 462 indivíduos.

A participação na pesquisa implicou em riscos mínimos aos participantes, conforme os parâmetros contidos na Resolução 466/12 do Conselho Nacional de Saúde/Ministério da Saúde, que dispõe sobre pesquisas envolvendo seres humanos. A pesquisa foi aprovada pelo comitê de ética (385.065/2013).

Utilizou-se o questionário validado denominado "Perfil do Estilo de Vida", proposto e publicado por Nahas, Barros e Francalacci (2000), acompanhado do Termo de Consentimento Livre e Esclarecido - TCLE em duas vias. O instrumento baseia-se no "Pentáculo do bem-estar" (NAHAS; BARROS; FRANCALACCI, 2000). Foram acrescidas perguntas de cunho epidemiológico e opiniões pessoais da amostra sobre o tema. O questionário tem sete seções: dados demográficos, opinião pessoal, características nutricionais, níveis de estresse, atividade física habitual, comportamentos sociais e comportamentos preventivos. 
Os itens do instrumento proposto validado foram estruturados com base no modelo de escala de Likert, onde havia quatro opções, as quais variavam de 0 a 3 , sendo 0 o ponto de menor concordância e 3 o de maior concordância. O desenvolvimento deste instrumento segue as normas de instrumentos internacionais conhecidos.

Cada seção conta com questões especificas sobre o tema e o participante respondeu conforme a escala de 0 a 3 , sendo:

(0) - absolutamente não faz parte do meu estilo de vida;

(1)- às vezes corresponde ao meu comportamento;

(2)- quase sempre verdadeiro no meu comportamento;

(3)- a afirmação é sempre verdadeira no meu dia-adia; faz parte do meu estilo de vida.

Os participantes que responderam com as alternativas "absolutamente não faz parte do meu estilo de vida" e "às vezes corresponde ao meu comportamento", foram arguidos do por que não adotaram ou praticam os hábitos de vida considerados os mais saudáveis. Dividiram-se essas respostas explicativas nos seguintes aspectos de análise:

1- Econômico: não há condição econômica atual para programar ou instituir as mudanças. 2- Cultural: não gostam, apoiam ou acreditam nas mudanças. 3- Temporal: relacionam problemas ligados à escassez de tempo e o estilo de vida atual. 4- Médico: estão impossibilitados de praticar alguma atividade ou de modificar o estilo de vida por orientação médica (problemas de saúde). 5 - Problemas com médicos: quase não vão ao médico, não são bem orientados pelos médicos. 6-Apoio:relatam falta de apoio dos familiares, amigos ou conhecidos. Ou alegam que cada um pensa de um jeito. 7- Outros: neste grupo se incluem todos os participantes que não se enquadram em nenhum dos outros motivos.

\subsection{Análise estatística}

Optou-se por uma modelo de amostragem não probabilística e a seleção dos indivíduos por conveniência com o controle de local, de forma a garantir a homogeneidade do ambiente e eliminar a interferência de possíveis variáveis aleatórias não analisadas no estudo.

As variáveis sob investigação foram divididas em dois grupos: quantitativas contínuas (idade) e qualitativas dicotômicas (gênero, raça, escolaridade, renda e região onde mora). Em seguida, foi realizada a análise estatística descritiva e exploratória dos dados utilizando frequências absolutas (n) e relativas (\%).
Para a análise comparativa das características das variáveis qualitativas dicotômicas, foram geradas tabelas de contingência $2 \mathrm{X} 2$ contendo as frequências absolutas (n) e relativas (\%). Para verificar a associação entre as variáveis foi realizado o Teste do QuiQuadrado de Independência (sem correção). O nível de significância para este teste é p-valor $\leq 0,05$ para um intervalo de confiança de $95 \%$.

A medida de associação utilizada foi o Odds Ratio de prevalência (OR de prevalência ou $\mathrm{RCP}=$ razão de chances prevalentes), que permite avaliar a relação entre a chance de um indivíduo exposto possuir a condição de interesse, quando comparada à do não exposto.

Visando facilitar a análise estatística, os dados foram organizados tematicamente, de forma a destacar os indicadores sociais considerados mais relevantes. Esses indicadores incluem: idade, sexo feminino ou masculino, cor, ganhar até três salários mínimos ou acima, e ter cursado até o ensino médio ou ter escolaridade superior.

Para o tratamento estatístico e montagem do banco de dados foi utilizado o Software estatístico SPSS Versão $15.0{ }^{\circledR}, 2010$.

\section{Resultados}

A amostra avaliada tem média de 36,6 $\pm 15,39$ anos e mediana de 33 anos. A distribuição dos indivíduos avaliados conforme gênero apresenta cerca de 54,5\% de mulheres e $45,5 \%$ de homens. Com relação à raça /cor declarada pelos indivíduos, a amostra possui predominância de brancos com $69,7 \% \%$, contra $30,3 \%$ de não brancos. Dentre os entrevistados, $60,4 \%$ estudaram até o ensino médio. Em relação à renda, $53,2 \%$ possuem renda menor que três salários mínimos.

Seguem abaixo, as tabelas que resumem os prinicipais resultados encontrados, demonstrando a relação entre as repostas às perguntas do questionário e as seguintes características: idade, sexo feminino ou masculino, cor, ganhar até três salários mínimos ou acima, e ter cursado até o ensino médio ou ter escolaridade superior. 


\section{TABela 1}

Associação da idade dos participantes com proposições a respeito do estilo de vida dos participantes

\begin{tabular}{|c|c|c|c|c|c|}
\hline Idade & (n) $100 \%$ & (\%) Proporção & p-valor & $\mathrm{RP}$ & $\mathrm{IC}-(\%)$ \\
\hline & \multicolumn{5}{|c|}{ Você evita ingerir alimentos gordurosos e/ou doces. } \\
\hline$\leq 33$ Anos & 234 & 46,2 & 0,001 & 1,866 & $1,282-2,688$ \\
\hline \multirow[t]{2}{*}{$\geq 34$ Anos } & 228 & 61,4 & & & \\
\hline & \multicolumn{5}{|c|}{ Ao menos duas vezes por semana você realiza exercícios que envolvam força e alongamento muscular. } \\
\hline$\leq 33$ Anos & 234 & 56,8 & 0,001 & 0,542 & $0,375-0,785$ \\
\hline \multirow[t]{2}{*}{$\geq 34$ Anos } & 228 & 41,7 & & & \\
\hline & \multicolumn{5}{|c|}{$\begin{array}{c}\text { Em seu dia a dia, você caminha ou pedala como meio de transporte e, preferencialmente, usa as escadas ao invés do } \\
\text { elevador. }\end{array}$} \\
\hline$\leq 33$ Anos & 234 & 61,1 & 0,001 & 0,543 & $0,375-0,786$ \\
\hline \multirow[t]{2}{*}{$\geq 34$ Anos } & 228 & 46,1 & & & \\
\hline & \multicolumn{5}{|c|}{ Não fuma e ingere álcool com moderação (menos de duas doses ao dia). } \\
\hline$\leq 33$ Anos & 234 & 71,8 & 0,055 & 1,513 & $0,985-2,323$ \\
\hline \multirow[t]{2}{*}{$\geq 34$ Anos } & 228 & 79,4 & & & \\
\hline & \multicolumn{5}{|c|}{ Seu lazer inclui reuniões com amigos, atividades esportivas em grupos, participação em associações. } \\
\hline$\leq 33$ Anos & 234 & 90,6 & 0,001 & 0,488 & $0,280-0,851$ \\
\hline \multirow[t]{2}{*}{$\geq 34$ Anos } & 228 & 82,5 & & & \\
\hline & \multicolumn{5}{|c|}{ Você procura cultivar amigos e está satisfeito com seus relacionamentos. } \\
\hline$\leq 33$ Anos & 234 & 90,6 & 0,001 & 0,360 & $0,212-0,611$ \\
\hline \multirow[t]{2}{*}{$\geq 34$ Anos } & 228 & 76,8 & & & \\
\hline & \multicolumn{5}{|c|}{ Você procura ser ativo em sua comunidade, sentindo-se útil no seu ambiente social. } \\
\hline$\leq 33$ Anos & 234 & 90,3 & 0,010 & 0,487 & $0,281-0,852$ \\
\hline$\geq 34$ Anos & 228 & 82,8 & & & \\
\hline
\end{tabular}

Legenda = n: número total da amostra; RP: razão de proporção; IC: intervalo de confiança.

Fonte: os autores (2015)

\section{TABEla 2}

Associação do sexo dos participantes com proposições a respeito do estilo de vida dos participantes

\begin{tabular}{|c|c|c|c|c|c|}
\hline Sexo & (n) $100 \%$ & (\%) Proporção & p-valor & $\mathrm{RP}$ & $\mathrm{IC}-(\%)$ \\
\hline & \multicolumn{5}{|c|}{ Suas noções sobre hábitos saudáveis se baseiam em orientações médicas. } \\
\hline Feminino & 252 & 59,5 & 0,040 & 0,680 & $0,470-0,984$ \\
\hline \multirow[t]{2}{*}{ Masculino } & 210 & 50,0 & & & \\
\hline & \multicolumn{5}{|c|}{ Sua alimentação diária inclui ao menos 5 porções de verduras e frutas. } \\
\hline Feminino & 252 & 67,5 & 0,007 & 0,595 & $0,408-0,869$ \\
\hline \multirow[t]{2}{*}{ Masculino } & 210 & 55,2 & & & \\
\hline & \multicolumn{5}{|c|}{$\begin{array}{l}\text { Ao menos duas vezes por semana você realiza exercícios que } \\
\text { envolvam força e alongamento muscular. }\end{array}$} \\
\hline Feminino & 252 & 38,9 & 0,001 & 2,554 & $1,753-3,721$ \\
\hline \multirow[t]{2}{*}{ Masculino } & 210 & 61,9 & & & \\
\hline & \multicolumn{5}{|c|}{$\begin{array}{c}\text { No seu dia a dia, você caminha ou pedala como meio de transporte e, preferencialmente, usa as escadas ao invés de } \\
\text { elevador. }\end{array}$} \\
\hline Feminino & 252 & 43,7 & 0,001 & 2,474 & $1,695-3,612$ \\
\hline \multirow[t]{2}{*}{ Masculino } & 210 & 65,7 & & & \\
\hline & \multicolumn{5}{|c|}{ Não fuma e ingere álcool com moderação(menos de duas doses ao dia). } \\
\hline Feminino & 252 & 81,0 & 0,003 & 0,525 & $0,342-0,806$ \\
\hline \multirow[t]{2}{*}{ Masculino } & 210 & 69,0 & & & \\
\hline & \multicolumn{5}{|c|}{ Discute sem alterar-se, mesmo quando contrariado. } \\
\hline Feminino & 252 & 55,2 & 0,001 & 1,986 & $1,347-2,926$ \\
\hline Masculino & 210 & 71,0 & & & \\
\hline
\end{tabular}

Legenda = n: número total da amostra; RP: razão de proporção; IC: intervalo de confiança.

Fonte: os autores (2015) 


\section{TABELA 3}

Associação da cor dos participantes com proposições a respeito do estilo de vida dos participantes

\begin{tabular}{lcc|c|c|c}
\hline Cor & (n) $100 \%$ & $(\%)$ Proporção & p-valor & RP & IC $-(\%)$ \\
\hline \multirow{2}{*}{ Branco } & \multicolumn{5}{c}{ Suas noções sobre hábitos saudáveis se baseiam em orientações médicas. } \\
Não Branco & 322 & 60,9 & 0,001 & 0,468 & $0,313-0,701$ \\
& 140 & 42,2 & & \\
Branco & 322 & Sua alimentação diária inclui ao menos 5 porções de verduras e frutas. \\
Não Branco & 140 & 58,7 & 0,031 & 1,587 & $1,041-2,421$ \\
\hline
\end{tabular}

Legenda = n: número total da amostra; RP: razão de proporção; IC: intervalo de confiança.

Fonte: os autores (2015)

\section{TABELA 4}

Associação do grau de escolaridade dos participantes com proposições a respeito do estilo de vida dos participantes

\begin{tabular}{|c|c|c|c|c|c|}
\hline Formação & (n) $100 \%$ & (\%) Proporção & p-valor & $\mathrm{RP}$ & $\mathrm{IC}-(\%)$ \\
\hline & \multicolumn{5}{|c|}{ Suas noções sobre hábitos saudáveis se baseiam em orientações médicas. } \\
\hline Até o fundamental & 279 & 49,8 & 0,004 & 1,744 & $1,191-2,554$ \\
\hline \multirow[t]{2}{*}{ Graduações mais altas } & 183 & 63,4 & & & \\
\hline & \multicolumn{5}{|c|}{ Você não fuma e ingere álcool com moderação (menos de 2 doses ao dia). } \\
\hline Até o fundamental & 279 & 72,0 & 0,051 & 1,557 & $0,999-2,439$ \\
\hline \multirow[t]{2}{*}{ Graduações mais altas } & 183 & 80,7 & & & \\
\hline & \multicolumn{5}{|c|}{$\begin{array}{c}\text { Você sempre usa cinto de segurança e, se dirige, o faz respeitando as normas de trânsito, nunca ingerindo álcool se vai } \\
\text { dirigir. }\end{array}$} \\
\hline Até o fundamental & 279 & 82,8 & 0,010 & 2,169 & $1,191-3,951$ \\
\hline \multirow[t]{2}{*}{ Graduações mais altas } & 183 & 91,3 & & & \\
\hline & \multicolumn{5}{|c|}{ Seu lazer NÃO inclui reuniões com amigos, atividades esportivas em grupos, participação em associações. } \\
\hline Até o fundamental & 279 & 17,2 & 0,003 & 2,508 & $1,339-4,698$ \\
\hline \multirow[t]{2}{*}{ Graduações mais altas } & 183 & 7,7 & & & \\
\hline & \multicolumn{5}{|c|}{ Você NÃO procura cultivar amigos e está satisfeito com seus relacionamentos. } \\
\hline Até o fundamental & 279 & 22,6 & 0,001 & 3,814 & $2,031-7,161$ \\
\hline Graduações mais altas & 183 & 7,1 & & & \\
\hline
\end{tabular}

Legenda = n: número total da amostra; RP: razão de proporção; IC: intervalo de confiança.

Fonte: os autores (2015)

Em relação aos demais dados coletados, quando avaliada a opinião pessoal, $55,2 \%$ da amostra tinham hábitos saudáveis baseados em orientações médicas, $93,7 \%$ acreditavam que mudar o estilo de vida poderia trazer benefícios à saúde, 69,5\% achavam que teriam apoio de seus amigos caso desejassem modificar um habito de vida e $92,2 \%$ imaginavam que os hábitos adotados no presente apresentariam repercussões no futuro.

Em relação à nutrição, $64,5 \%$ da amostra relataram que fazem 04 a 05 refeições variadas ao dia; os demais que não seguiam essa recomendação apresentaram como principais dificuldades: problemas não especificados (11\%), problemas culturais $(10,2 \%)$ e falta de tempo (9,7\%). Observamos que $61,9 \%$ da amostra incluíam 05 porções de verduras e frutas na alimentação diária e 53,7\% evitavam ingerir alimentos gordurosos e/ou doces, dentre os entrevistados que não adotavam estes hábitos alimentares, a principal justificativa foi a questão cultural $(18,4 \%$ e $28,6 \%$, respectivamente).

Quanto à atividade física, 51,5\% não realizam atividades físicas moderadas/intensas, de forma continua ou acumulada por 30 minutos 5 ou mais dias por semana, devido à questão cultural $(31,4 \%)$ e à falta de tempo (10,8\%). Notamos que 50,7\% não faziam exercícios que envolvessem força e alongamento muscular ao menos duas vezes por semana e as principais justificativas para isso também foram a falta de tempo e a questão cultural (22,9\% e $14,1 \%$, respectivamente). A maioria da amostra $(53,6 \%)$ caminhava ou pedalava como meio de transporte e preferencialmente usava as escadas ao invés do elevador. Os que não seguiam essas rotinas, 


\section{TABela 5}

Associação da renda dos participantes com proposições a respeito do estilo de vida dos participantes

\begin{tabular}{|c|c|c|c|c|c|}
\hline Renda (referente ao salário minimo ) & (n) $100 \%$ & $(\%)$ Proporção & p-valor & $\mathrm{RP}$ & $\mathrm{IC}-(\%)$ \\
\hline & \multicolumn{5}{|c|}{ Suas noções sobre hábitos saudáveis se baseiam em orientações médicas. } \\
\hline Até 03 salários & 246 & 44,7 & 0,001 & 2,525 & $1,728-3,689$ \\
\hline \multirow[t]{2}{*}{4 Ou mais salários } & 216 & 67,1 & & & \\
\hline & \multicolumn{5}{|c|}{ Se você desejasse modificar um hábito de vida, você acha que teria apoio dos seus amigos. } \\
\hline Até 03 salários & 246 & 65,4 & 0,045 & 1,508 & $1,009-2,255$ \\
\hline \multirow[t]{2}{*}{4 Ou mais salários } & 216 & 74,1 & & & \\
\hline & \multicolumn{5}{|c|}{ Você faz 4 a 5 refeições variadas ao dia, incluindo café da manhã completo. } \\
\hline Até 03 salários & 246 & 60,2 & 0,038 & 1,505 & $1,023-2,214$ \\
\hline \multirow[t]{2}{*}{4 Ou mais salários } & 216 & 69,4 & & & \\
\hline & \multicolumn{5}{|c|}{$\begin{array}{l}\text { Ao menos duas vezes por semana você realiza exercícios que } \\
\text { envolvam força e alongamento muscular. }\end{array}$} \\
\hline Até 03 salários & 246 & 43,9 & 0,012 & 1,597 & $1,105-2,308$ \\
\hline \multirow[t]{2}{*}{4 Ou mais salários } & 216 & 55,6 & & & \\
\hline & \multicolumn{5}{|c|}{$\begin{array}{l}\text { No seu dia a dia, você caminha ou pedala como meio de transporte e, preferencialmente, usa as } \\
\text { escadas ao invés de elevador. }\end{array}$} \\
\hline Até 03 salários & 246 & 49,6 & 0,050 & 1,423 & $1,099-2,057$ \\
\hline \multirow[t]{2}{*}{4 Ou mais salários } & 216 & 58,3 & & & \\
\hline & \multicolumn{5}{|c|}{ Não fuma e ingere álcool com moderação (menos de duas doses ao dia). } \\
\hline Até 03 salários & 246 & 71,0 & 0,019 & 1,681 & $1,087-2,598$ \\
\hline \multirow[t]{2}{*}{4 Ou mais salários } & 216 & 80,7 & & & \\
\hline & \multicolumn{5}{|c|}{$\begin{array}{c}\text { Você sempre usa cinto de segurança e, se dirige,o faz respeitando as normas de trânsito, nunca } \\
\text { ingerindo álcool se vai dirigir. }\end{array}$} \\
\hline Até 03 salários & 246 & 82,0 & 0,016 & 1,967 & $1,126-3,435$ \\
\hline \multirow[t]{2}{*}{4 Ou mais salários } & 216 & 90,8 & & & \\
\hline & \multicolumn{5}{|c|}{ Seu lazer inclui reuniões com amigos, atividades esportivas em grupos, participação em associações. } \\
\hline Até 03 salários & 246 & 83,0 & 0,029 & 1,857 & $1,059-3,255$ \\
\hline \multirow[t]{2}{*}{4 Ou mais salários } & 216 & 90,6 & & & \\
\hline & \multicolumn{5}{|c|}{ Você mantém uma discussão sem se alterar, mesmo quando contrariado } \\
\hline Até 03 salários & 246 & 58,1 & 0,046 & 1,471 & $1,005-2,152$ \\
\hline 4 Ou mais salários & 216 & 67,1 & & & \\
\hline
\end{tabular}

Legenda = n: número total da amostra; RP: razão de proporção; IC: intervalo de confiança.

Fonte: os autores (2015)

$18,8 \%$ alegavam falta de tempo e $15,6 \%$ problemas culturais.

Em relação ao comportamento preventivo ao praticar hábitos saudáveis, a maioria $(67,1 \%)$ conhece seus níveis de pressão arterial e de colesterol e procura controlá-los e os que não conhecem, apresentaram como principal justificativa a falta de tempo $(10,4 \%)$. A maior parte da amostra $(75,5 \%)$ não fuma e ingere álcool com moderação (menos de duas doses ao dia); entre os que fumam e bebem, a principal razão citada foram os problemas médicos (12,8\%). Além disso, a maioria $(86,2 \%)$ usa cinto de segurança e, ao dirigir, o faz respeitando as normas de trânsito, nunca ingerindo álcool; os que não adotam essas medidas preventivas alegaram como principal motivo a questão cultural $(9,3 \%)$.
Quanto ao relacionamento social dos entrevistados, 86,6\% incluem em seu lazer reuniões com amigos, atividades esportivas em grupos e participação em associações; 83,5\% procuram cultivar amigos e estão satisfeitos com os seus relacionamentos e 86,6\% procuram ser ativos em sua comunidade, sentindo-se úteis no seu ambiente social.

A respeito do controle de estresse, 83,5\% reservam tempo (ao menos 5 minutos) todos os dias para relaxar; $74,7 \%$ equilibram o tempo dedicado ao trabalho e ao lazer e $62,3 \%$ se mantém numa discussão sem se alterar. As principais razões para aqueles que tem dificuldades no controle do estresse foram falta de tempo $(6,1 \%, 17,1 \%$ e $16,7 \%$, respectivamente $)$ e questão cultural $(5,8 \% ; 3,5 \%$ e $10 \%)$. 


\section{Discussão}

Para um individuo mudar seu estilo de vida e agregar valor em saúde, é necessário que tenha inicialmente plena consciência dos hábitos saudáveis e que aceite e busque ativamente essa transformação. $\mathrm{O}$ simples conhecimento e entendimento das inúmeras vantagens dos bons hábitos não necessariamente fazem com que as pessoas adotem tempestivamente os bons costumes. Dentre os obstáculos existentes para que ocorra uma melhora da qualidade de vida, a falta de tempo e de espaço adequado para realizar alguma atividade física e recursos financeiros insuficientes faz parte das principais queixas dos indivíduos que não adotam boas práticas (GAUDÊNCIO, 1999).

Ser mulher, branca, ter escolaridade mais elevada e receber quatro ou mais salários mínimos tem relação significativa com o fato de as noções sobre hábitos saudáveis se basearem em orientações médicas, o que pode ser notado pela análise em conjunto da tabela 2 (sexo) com p-valor 0,04 (IC 0,470-0,984), tabela 3 (cor) com p-valor 0,001 (IC 0,313-0,701), tabela 4 (formação) com p-valor 0,004 (IC 1,191-2,554) e tabela 5 (renda) com p-valor 0,001 (IC 1,728-3,686). Além disso, receber um salário maior implica em maior chance de acreditar que teria apoio dos amigos ao modificar um hábito com p-valor 0,045 e IC 1,009 $-2,255$, vide tabela 5 .

Dentre as razões de se escolher um determinado tipo de nutrição, o custo dos alimentos está acima do conhecimento nutricional ou motivação para o consumo. Os hábitos alimentares se inserem em estruturas culturais, econômicas e políticas, sendo necessário dar maior ênfase na realização de estratégias direcionadas para o aumento do consumo de frutas, legumes e verduras (NEUTZLING, 2009; UNITED STATES DEPARTMENT OF AGRICULTURE [USDS], 2014).

Em nossa pesquisa, observamos que receber quatro ou mais salários mínimos tem relação significativa com o fato de fazer quatro a cinco refeições variadas ao dia, incluindo café da manha completo, com p-valor 0,038 (IC 1,023-2,214 vide tabela 5. Sob outra ótica, outros autores analisaram que em famílias de baixa renda, a proporção das despesas com alimentação é maior do que em famílias de maior renda. Tal raciocínio acompanha, similarmente, o que ocorre em comparação entre países considerados como desenvolvidos e aqueles em desenvolvimento (KILSZTAJN, 1998).

Em relação aos participantes maiores de 34 anos, houve relação significativa com o fato de evitar ingerir alimentos gordurosos com p-valor 0,001 (IC 1,282-2,688 vide tabela 01 . Nas últimas décadas, o avanço da industrialização e as mudanças culturais proporcionaram o surgimento de numerosos alimentos industrializados ricos em gordura, açúcar e sal, que são de fácil acesso e ser relacionam diretamente com o curto tempo despendido para a alimentação, principalmente em centros urbanos (FIGUEIREDO; JAIME; MONTEIRO, 2008; MOREIRA; SANTOS; LOPES, 2012; LOBATO; COSTA; SICHIERI, 2009). Encontramos associação significativa do fato de ser mulher e branca em relação a incluir ao menos 5 porções de verduras e frutas (com p-valores respectivos de 0,007 e 0,031 ), vide tabelas 2 e 3 . Outro estudo (MONDINI, 2010), com indivíduos maiores de 30 anos, evidenciou que as mulheres consumiam mais frutas e hortaliças quando comparadas aos homens e apontou que esse consumo estava também diretamente relacionado com o aumento na idade e do nível socioeconômico, mostrando ser maior com a idade e renda mais elevada (MONDINI, 2010). Além disso, estudos apontam que o consumo de hortaliças e frutas se faz maior com o aumento na idade e nível socioeconômico (DEON et al., 2015; MALTA; PAPINI; CORRENTE, 2013).

Quanto ao item atividade física, nosso estudo evidenciou que a maioria dos indivíduos entrevistados realiza pelo menos 30 minutos de atividades físicas moderadas/intensas, de forma contínua ou acumulada, 5 ou mais dias por semana. Mais da metade dos participantes, ao menos duas vezes por semana, realiza exercícios que envolvam força e alongamento muscular, caminha ou pedala como meio de transporte e, preferencialmente, usa as escadas ao invés de elevador no dia a dia. Esse resultado guarda relação com outros estudos nos quais se observou maior prevalência de pessoas ativas na população em geral (BARROS; IAOCHITE, 2012; JUNIOR et al, 2011).

Em nossa pesquisa houve relação significativa entre ser homem, receber mais de 3 salários e ter menos de 33 anos, com fazer exercícios que envolvam força e alongamento muscular de forma mais frequente, caminhar ou pedalar como meio de transporte e preferencialmente usar as escadas ao invés do elevador (vide tabelas 1,2 e 5 , com respectivos p-valores de 0,$001 ; 0,001$ e 0,012 ). De forma semelhante, outros autores evidenciaram na população adulta que a prevalência de atividade física no lazer é maior em homens mais jovens e de maior renda (NASSER et al., 2016). Em relação à idade há influência no uso ou não da bicicleta, uma vez que as faixas etárias têm características intrínsecas relacionadas com a habilidade motora e condicionamento físico para pedalar, e idades mais avançadas poderiam limitar o uso desse veículo (PUCHER; BUEHLER, 2008; SOUZA, P. B., 2013).

HU Revista, Juiz de Fora, v. 43, n. 1, p. 51-60, jan./jun. 2017 
Em relação à renda, um estudo avaliou significância entre o nível de atividade física e as características sóciodemográficas dos usuários, referindo que a possível explicação para esse acontecimento pode estar relacionada ao fato de que, com menor grau de instrução, os indivíduos têm menos consciência da importância de adotar atividades físicas como forma de lazer e para manterem-se saudáveis. A explicação para a prática de atividade física insuficiente nos ambientes públicos é que a baixa renda e escolaridade estão possivelmente implicadas em menor qualificação profissional e, consequentemente, em funções mais árduas e fisicamente desgastantes no trabalho, com consequente cansaço físico e menor vitalidade no tempo de lazer" (BARROS; NAHAS, 2001; COSTA, 2009).

Fatores culturais e sociais parecem determinar algumas diferenças com relação à prática de atividade física de acordo com o sexo: os homens, em sua maioria, associam os exercícios físicos ao prazer, enquanto as mulheres praticam atividade física por questões de saúde, orientação médica ou por estética. Ainda, os homens se envolvem mais em atividades em grupos, como a prática de esportes coletivos, enquanto as mulheres preferem atividades individuais como caminhar, correr e andar de bicicleta (MARTINS, 2000; MONTEIRO et al., 2003).

Observamos em nossa pesquisa o seguinte perfil: ser mulher ( $\mathrm{p}$-valor 0,003 ), tabela 2 , e receber quatro ou mais salários mínimos (p-valor de 0,019), tabela 5 , tem relação significativa com o fato de não fumar e ingerir álcool com moderação (menos de duas doses ao dia), concordante com vários outros estudos. Pesquisadores revelaram que a prevalência de fumo entre os homens brasileiros foi maior quando comparada com as mulheres brasileiras e que o consumo diário de cigarros guarda relação inversa com a renda, ou seja, quanto maior a renda menor o consumo de cigarros (BARROS, A. J. D. et al., 2011). Outro estudo apontou que em relação à escolaridade, para ambos os sexos, a prevalência de tabagismo é tanto menor quanto maior for o nível de graduação (BERTO; CARVALHAES; MOURA, 2010). Com relação ao álcool, outra pesquisa evidenciou que o maior consumo alcoólico ocorre no grupo dos homens, idosos, negros ou pardos, com renda familiar mais baixa (COSTA et al., 2004).

$\mathrm{O}$ fato de a pessoa ter qualificação escolar maior e receber quatro ou mais salários mínimos tem relação significante com a ocorrência de usar cinto de segurança e, ao dirigir, o faz respeitando as normas de transito, nunca ingerindo álcool ( $\mathrm{p}$-valores de 0,01 e 0,017 , respectivamente, encontrados nas tabelas 4 e 5).
O relacionamento social é diretamente conexo com a satisfação da vida pessoal e/ou à qualidade de vida do indivíduo. Houve associação significativa entre indivíduos que têm menos de 34 anos e escolaridade mais elevada com o fato de o lazer incluir reuniões com amigos, atividades esportivas em grupos, participação em associações, cultivo de amizades e alto grau de satisfação com seus relacionamentos, sendo a idade observada na tabela 1 , com p-valores $0,001,0,001$ e 0,010 , respectivamente, e a escolaridade observada na tabela 4, com p-valores de 0,003 e 0,001, respectivamente, para os itens analisados. Avaliando adultos e idosos, outros autores evidenciaram satisfação entre os respondentes com seus relacionamentos (RESENDE et al., 2006). Pesquisadores notaram que as mulheres estão expostas em maior proporção à percepção de estresse elevado (talvez devido à dupla jornada de trabalho e obrigações domésticas); e àquelas com maior nível econômico foram associadas à menor prevalência da percepção de níveis elevados de estresse (BARROS; NAHAS., 2001). Em nosso estudo houve uma associação significativa do sexo feminino com a capacidade de se alterar em uma discussão quando contrariado(p valor de 0,001 , tabela 2) e ter renda inferior a quatro salários ( $\mathrm{p}$-valor de 0,046 ,tabela 5)

O estudo apresenta limitações por ser um estudo transversal, no qual há impossibilidade de se estabelecer prova causal. Além disso, o uso de questionários requer motivação e disposição dos participantes de responder às perguntas e a confiabilidade das informações dos questionários é dependente da veracidade das informações fornecidas e capacidade de compreensão dos participantes.

\section{Conclusão}

As principais dificuldades encontradas para se ter um estilo de vida adequado foram ligadas a fatores culturais, ou seja, vinculadas às crenças quanto às possibilidades/efetividades das mudanças; o segundo motivo mais importante foi a falta de tempo. A amostra em geral busca manter um estilo de vida saudável, pois a maioria tem noções dos hábitos saudáveis com base nas orientações médicas. Também mantém hábitos nutricionais bem satisfatórios, realizando algum tipo de atividade física, buscando manter os cuidados com a própria saúde, desenvolvendo bom relacionamento social e controlando o stress. O perfil que melhor cuida da própria saúde compreende o grupo que tem nível de escolaridade mais elevado, com menos de 34 anos e que recebe quatro ou mais salários mínimos por mês, não importando o sexo.

Conhecer o perfil da população ao seu redor permite estabelecer novas práticas de abordagem e de 
estimulo aos pacientes em relação à adoção de hábitos mais saudáveis, permitindo a avaliação das principais limitações relacionadas a mudança de estilo de vida.

\title{
Attitudes and the knowledge about healthy lifestyle practices in a population-based sample of Juiz de Fora
}

\begin{abstract}
The new welfare pentacle refers to five characteristics of the life style that support the individual health promotion: stress levels, nutritional characteristics, the usual physical activities, preventive behavior and the quality of human relationships. As we realize how difficult it is to change the lifestyle of patients and act positively on preventing diseases - or treating them -, we have sought to identify the obstacles in switching to a healthy lifestyle, besides evaluating habits of life and knowledge about healthy practices. Thereby, we have elaborated a cross-sectional, descriptive and quantitative study held in the city of Juiz de Fora-MG, and data were collected from a validated questionnaire, the new welfare pentacle, comprising a sample of 462 individuals. Most interviewees have notions of healthy habits, $55,2 \%$ of them based on medical guidance; $93,7 \%$ believe in the benefits of the life style changes; $92,2 \%$ presume that current healthy habits reverberate on the future and 53,6\% practice some kind of physical activity. In general, they keep satisfactory nutritional habits, $61,9 \%$ reported daily intake of 05 portions of vegetables and 53,7\% avoid greasy food and/or treats. The difficulties were mainly found to be associated to cultural factors, linked to beliefs. As for the possibilities/effectiveness of changes, lack of time was the second greatest difficulty found. Finally, it was concluded that the profile that best takes care of one's own health includes those with a higher educational level, under 34 years of age and who receive four or more minimum wages per month, regardless of sex.
\end{abstract}

Keywords Life style. Health. Physical activity.

\section{REFERÊNCIAS}

BARROS, A. J. D. et al. Tabagismo no Brasil: desigualdades regionais e prevalência segundo características ocupacionais. Revista Ciência \& Saúde Coletiva, Rio de Janeiro, v. 16, n. 9, p.3707-3716, set. 2011.

BARROS, M. B.; IAOCHITE, R. T. Autoeficácia para a prática de atividade física por indivíduos adultos. Revista Motricidade, Vila Real, vol. 8, n. 2. p. 32-41, abr-jun. 2012.

BARROS, M. V. G; NAHAS, M. V. Comportamentos de risco, auto-avaliação do nível de saúde e percepção de estresse entre trabalhadores da indústria. Revista de Saúde Pública, São Paulo, v. 35, n. 6, p. 554-563, dez. 2001.

BAUM, F.; FISHER, M. Why behavioral health promotion endures despite its failure to reduce health inequities. Sociology of Health \& Illness, Hoboken, v. 36, n. 2, p. 213-225, fev. 2014.

BERTO, S. J. P.; CARVALHAES, M. A. B. L; MOURA, E. C. Tabagismo associado a outros fatores comportamentais de risco de doenças e agravos crônicos não transmissíveis. Caderno de Saúde Pública, Rio de Janeiro, v. 26, n. 8, p. 1573-1582, ago. 2010.

BUSS, P. M. Promoção da Saúde da Família. Revista Brasileira de Saúde da Família, Brasília, ano II, n. 6, p. 50-63, dez. 2002.
COSTA, J. S. D. et al. Consumo abusivo de álcool e fatores associados: estudo de base populacional. Revista de Saúde Pública, São Paulo, v. 38, n. 2, p. 284-291, abr. 2004.

COSTA, Evelyn Fabiana. Prática de atividade física e sua relação com a escolaridade em adultos de Ermelino Matarazzo, Zona Leste de São Paulo, SP. 2009. Dissertação (Mestrado em Nutrição em Saúde Pública) - Faculdade de Saúde Pública, Universidade de São Paulo, São Paulo, 2009.

DEON, R. G. et. al. Consumo de alimentos dos grupos que compõem a pirâmide alimentar americana por idosos brasileiros: uma revisão. Revista Ciência \& Saúde, Porto Alegre, v. 8, n. 1, p. 26-34, jan.-abr. 2015.

DOSSE, C. et al. Fatores associados à não adesão dos pacientes ao tratamento de Hipertensão Arterial. Revista Latino-Americana de Enfermagem, Ribeirão Preto, v. 17, n 2, p. 201-206, mar-abr. 2009.

FIGUEIREDO, I. C. R; JAIME, P. C.; MONTEIRO, C. A. Fatores associados ao consumo de frutas, legumes e verduras em adultos da cidade de São Paulo. Revista de Saúde Pública, São Paulo, v. 42, n. 5, p 777-785, ago. 2008.

GAUDÊNCIO, P. Mudar e vencer: como as mudanças podem beneficiar pessoas e empresas. São Paulo: Editora Gente, 1999.

HALLAL, P. C. et al. Global physical activity levels: surveillance progress, pitfalls, and prospects. The Lancet, Reino Unido, v. 380, p. 247-257, 21 jul. 2012.

HU Revista, Juiz de Fora, v. 43, n. 1, p. 51-60, jan./jun. 2017 
JUNGUES, C. F. Influência da cultura no comportamento alimentar em gestantes: contribuições para enfermagem. 107 f. Dissertação (Mestrado em Enfermagem), Faculdade de Enfermagem, Universidade Federal de Santa Maria, Santa Maria, 2010.

JUNIOR, J. S. M. et al. Nível de Atividade Física e Perfil Sociodemográfico dos Usuários dos Ambientes Públicos de Atividades Físicas na Cidade de João Pessoa-PB. Revista Brasileira de Ciências da Saúde, João Pessoa, v. 15, n. 3, p. 349-356, 2011.

KILSZTAJN, S. Padrão de alimentação e distribuição de renda no Brasil. Pesquisa \& Debate, São Paulo, v.9, n. 2, p. 44-86, 1998.

LOBATO, J. C. P.; COSTA, A. J. L.; SICHIERI, R. Food intake and prevalence of obesity in Brazil: an ecological analysis. Public Health Nutrition, San Luis Obispo, v. 12, n. 11, p. 2209-2215, nov. 2009.

MALTA, M. B.; PAPINI, S. J.; CORRENTE, J. E. Avaliação da alimentação de idosos de município paulista: aplicação do Índice de Alimentação Saudável. Revista Ciência \& Saúde Coletiva, Rio de Janeiro, v.18, n. 2, p. 377-384, fev. 2013.

MARTINS, M. O. Estudo dos fatores determinantes da prática de atividades físicas de professores universitários. 183 f. Dissertação (Mestrado em Atividade Física e Saúde - CDS), Faculdade de Educação Física, Universidade Federal de Santa Catarina, Florianópolis, 2000.

MONDINI, L. et al. Consumo de frutas e hortaliças por adultos em Ribeirão Preto, SP. Revista de Saúde Pública, São Paulo, v. 44, n. 4, p. 686-694, ago. 2010.

MONTEIRO, C. A. et al. A descriptive epidemiology of leisuretime physical activity in Brazil, 1996-1997. Revista Panamericana de Salud Publica, Washington, v. 14, n. 4, p. 246-254, out. 2003. MOREIRA, R. A. M.; SANTOS, L. C.; LOPES, A. C. S. A qualidade da dieta de usuários de serviço de promoção da saúde difere segundo o comportamento alimentar obtido pelo modelo transteórico. Revista Nutrição, Campinas, v. 25, n. 6, p. 719-730, dez. 2012.

\section{NAHAS, M. V.; BARROS, M. V. G.;FRANCALACCI, V. O}

pentáculo do bem-estar - Base conceitual para avaliação do estilo de vida de indivíduos ou grupos. Revista Brasileira de Atividade Física \& Saúde, Pelotas, v. 5, n. 2, p. 48-59, 2000.

NASSER, Roberta Lacerda et al. Atividade física de lazer e uso de substâncias lícitas em uma amostra populacional de adultos jovens. Revista Ciência \& Saúde Coletiva, Rio de Janeiro, v. 21, n. 1, p. 63-70, jan. 2016.
NEUTZLING, M. B. et al. Fatores associados ao consumo de frutas, legumes e verduras em adultos de uma cidade no Sul do Brasil. Caderno de Saúde Pública, Rio de Janeiro, v. 25, n. 11, p. 2365-2374, nov. 2009.

PUCHER, J.; BUEHLER, R. (2008). Cycling for Everyone: Lessons from Europe. Transportation Research Board, CDROM 87th Annual Meeting, Washington, D.C.

RESENDE, M. C. et al. Rede de relações sociais e satisfação com a vida de adultos e idosos. Revista Psicologia para América Latina, México, n.5, fev. 2006.

RICOU, M. et al. A comunicação e a alteração de comportamentos. Revista Psicologia, Saúde \& Doenças, Lisboa, v. 5 , n. 2, p. 131-144, nov. 2004.

SALLIS, J. F.; OWEN, N. Physical activity \& behavioral medicine. Sage Publications, Thousand Oaks, v. 3, p. 110-134, 1999.

SILVA, R. S. et al. Atividade física e qualidade de vida. Revista Ciência \& Saúde Coletiva, Rio de Janeiro, v. 15, n. 1, p. 115-120, jan. 2010.

SOUZA, C. A. et al. Prevalência de atividade física no lazer e fatores associados: estudo de base populacional em São Paulo, Brasil, 2008-2009. Caderno de Saúde Pública, Rio de Janeiro, v. 29, n. 2, p. 270-282, fev. 2013.

SOUZA, P. B. Análise de fatores que influem no uso da bicicleta para fins de planejamento cicloviário. $190 \mathrm{f}$. Tese (Doutorado - Programa de pós-graduação em Engenharia de Transporte e Área de Concentração em Planejamento e Operação de Sistemas de Transportes), Escola de Engenharia de São Carlos, Universidade de São Paulo, São Carlos, 2012.

\section{UNITED STATES DEPARTMENT OF AGRICULTURE}

[USDS]. Economic Research Service [ERS]. USDA calculations based on annual household expenditure data from ERS: Percent of consumer expenditures spent on food, alcoholic beverages, and tobacco that were consumed at home, by selected countries, 2014. Washington, 2014.

VASCONCELOS, K. E. L.; SCHMALLER, V. P. V. (Nova) promoção da saúde: configurações no debate do serviço social. Emancipação, Ponta Grossa, v. 14, n. 1, p. 129-146, 2014.

WORLD HEALTH ORGANIZATION. Preamble to the Constitution of the World Health Organization as adopted by the International Health Conference, New York, 19-22 June, 1946; signed on 22 July 1946 by the representatives of 61 States (Official Records of the World Health Organization, no. 2, p. 100) and entered into force on 7 April 1948. Disponível em:. < http://www.who.int/about/definition/en/print.html>. Acesso em: 22/04/2016

Enviado em 15/05/2016 